\title{
Overexpression of fibroblast growth factor receptor 4 in high-grade pancreatic intraepithelial neoplasia and pancreatic ductal adenocarcinoma
}

\author{
NORIO MOTODA ${ }^{1}$, YOKO MATSUDA ${ }^{1}$, MUNEHIKO ONDA $^{1}$, \\ TOSHIYUKI ISHIWATA ${ }^{1}$, EIJI UCHIDA ${ }^{2}$ and ZENYA NAITO ${ }^{1}$ \\ ${ }^{1}$ Department of Pathology, Integrative Oncological Pathology, Graduate School of Medicine, \\ Nippon Medical School, 1-1-5 Sendagi, Bunkyo-ku, Tokyo 113-8602; ${ }^{2}$ Surgery for Organ and Biological Regulation, \\ Graduate School of Medicine, Nippon Medical School, 1-1-5 Sendagi, Bunkyo-ku, Tokyo 113-8603, Japan
}

Received August 28, 2010; Accepted October 26, 2010

DOI: 10.3892/ijo_00000832

\begin{abstract}
The overexpression of fibroblast growth factor receptor (FGFR) 4 has been reported in various human cancers, but it has not been studied in pancreatic ductal adenocarcinoma (PDAC) or its precursor lesion, pancreatic intraepithelial neoplasia (PanIN). Moreover, there is controversy as to whether FGFR4 has a mitogenic role in carcinogenesis or other functions. Therefore, the expression and roles of FGFR4 in pancreatic cancer were investigated. Immunohistochemical staining was performed using an anti-FGFR4 antibody in PDAC and PanIN cases. The expression levels of FGFR4 mRNA and protein were investigated in PDAC cell lines by qRT-PCR and Western blot, respectively. Changes were analyzed in cell morphology, proliferation, migration, invasion and attachment in PDAC cell lines with or without the stimulation of FGFR4 by FGF19, as a known specific ligand. The changes in mRNA levels associated with transformation and tumorigenesis as a result of FGF19 administration were also evaluated. FGFR4 was expressed in 39 of 53 PDAC cases $(73.6 \%)$ and its expression tended to be related to longer overall survival $(\mathrm{P}=0.068)$. Moreover, it was frequently expressed in high-grade PanIN lesions [10 of 11 lesions $(90.9 \%)$ ], whereas it was hardly expressed in lowgrade PanIN lesions [1 of 10 lesions $(10.0 \%)$ ] $(\mathrm{P}=0.0003)$. FGFR4 stimulation of PDAC cells resulted in significantly increased cell adhesion to laminin and fibronectin $(\mathrm{P}<0.05)$ and decreased cell migration $(\mathrm{P}<0.05)$. The results of PCR array analysis indicated that this was a result of up-regulation of the integrin $\alpha 4$ family. In contrast, cell morphology or
\end{abstract}

Correspondence to: Dr Zenya Naito, Department of Pathology, Integrative Oncological Pathology, Nippon Medical School, 1-1-5 Sendagi, Bunkyo-ku, Tokyo 113-8602, Japan

E-mail: naito@nms.ac.jp

Key words: fibroblast growth factor receptor 4 (FGFR4), fibroblast growth factor 19 (FGF19), pancreatic intraepithelial neoplasia, pancreatic ductal adenocarcinoma, cell adhesion proliferation in PDAC cells was not affected. We showed that FGFR4 expression is markedly increased in high-grade PanIN and PDAC compared with that in normal and lowgrade PanIN, and that FGFR4 stimulation by FGF19 of PDAC cells contributes to tumor suppression by increasing cell adhesion to extracellular matrix.

\section{Introduction}

Pancreatic cancer is the fourth leading cause of cancer death in the United States and the fifth in Japan $(1,2)$. Five-year survival remains $<10 \%$ despite the development of therapeutics and the excision rate is $<40 \%$ in Japan (2). The reason for the poor prognosis of pancreatic cancer is that it is often diagnosed at too late a stage to operate radically. The patients suffer poor quality of life such as bile obstruction, digestive stenosis, severe pain and endocrine disorder. Moreover, the patients who undergo resection frequently exhibit disease recurrence with metastasis and peritoneal dissemination. Most pancreatic cancer is diagnosed as pancreatic ductal adenocarcinoma (PDAC), and it is considered that PDAC shows a ductal phenotype and involves a precursor intraductal lesion, called pancreatic intraepithelial neoplasia (PanIN) $(1,2)$. PanIN lesions are divided into three grades, PanIN 1, 2 and 3, which are represented as hyperplasia, moderate dysplasia, and severe dysplasia/carcinoma in situ, respectively (3). Recently, the molecular mechanism of pancreatic cancer has become clearer. The K-ras mutation is present in up to $90 \%$ of PDAC, resulting in activation of mitogen-activated protein kinase (MAPK) cascade. Loss of heterozygosity of tumor suppressor genes, such as p16 (CDKN-2A), p53 and deleted in pancreatic carcinoma locus 4 (DPC4, also known as SMAD4), is observed at a high frequency (4). These genetic alterations of PDAC also occur in PanIN lesions with a high frequency and are associated with the stage of PanIN grading. Molecular target therapy has been gradually developed, but the intended effects are not obtained sufficiently often (5).

The roles of fibroblast growth factor (FGF) and its receptor (FGFR) have been reported for carcinogenesis in various organs. The FGFR family consists of FGFR 1-5 and, with the 
exception of FGFR5, also known as fibroblast growth factor receptor-like 1 (FGFRL1), these members have a common characteristic structure of three extracellular immunoglobulin (Ig) domains, a single-pass transmembrane domain and a cytoplasmic tyrosine kinase domain (6). FGFR4 is one of the members of the FGFR family, and FGF1, 2, 4, 6, 8, 9, 16, 17, 18 and 19 have affinity to the receptor (6-8). Interestingly, FGFR4 has no splicing variants in Ig domain III, unlike the other FGFRs, and FGF19 binds specifically to FGFR4 (7). FGFR4 is overexpressed in hepatocellular carcinoma (HCC) (9), breast cancer (10), renal cell carcinoma (11) and malignant melanoma (12), but its role in oncogenesis is controversial; in hepatocellular carcinoma, some researchers have indicated that the activation of FGFR4 is related to increases in cell migration and $\alpha$-fetoprotein (AFP) production as well as worse prognosis (9), while others have stated that overexpression of FGFR4 is related to an increase in apoptosis and better prognosis (13).

FGF19 is a member of the FGF19 subfamily (FGF19, 21 and 23) and is known as a specific ligand for FGFR4 (7). It has anti-diabetic effects independently of known substances (14) and mediates bile acid homeostasis in the liver by inducing cholesterol 7- $\alpha$-hydroxylase (CYP7A1) repression (15). The FGF19 subfamily has some unique features. First, it behaves in endocrine fashion due to a disrupted heparin binding motif, in contrast to other FGF subfamilies (16). Second, it has been thought to require a co-receptor, ßKlotho, for binding to FGFRs (16); however, in a recent study, this unique FGF was found to bind only to FGFR4 lacking BKlotho protein in the presence of heparin (17). In the field of oncology, it has been reported that FGF19 is expressed in colonic adenocarcinoma (18) and hepatocellular carcinoma (19). The stimulation by FGF19 is considered to induce the ß-catenin signaling pathway and be related to cancer progression $(18,20)$. However, the role of FGFR4 and FGF19 in pancreatic carcinogenesis has not been clearly determined.

In the present study, in order to determine the expression and roles of FGFR4 in PDAC, we carried out immunohistochemical analysis of FGFR4 in each phase of pancreatic carcinogenesis, including normal pancreas, low- and highgrade PanIN and PDAC cases. We report that FGFR4 is expressed in both PDAC and high-grade PanIN lesions at a high frequency. We also show novel functions of FGF19/ FGFR4 signaling, namely, increased cell adhesion and decreased cell migration as a result of up-regulation of the integrin family.

\section{Material and methods}

Material. The following were purchased: isogen from Nippon Gene (Tokyo, Japan); LightCycler 480 Probe Master and Universal Probe Library nos. 70 and 72 from Roche Diagnostics (Mannheim, Germany); High Capacity cDNA reverse transcription kit from Applied Biosystems, Inc. (Carlsbad, CA); FastPure RNA kit from Takara Biotech (Tokyo, Japan); M-PER mammalian protein extraction reagent and Super Signal West Dura Extended Duration chemiluminescent substrates from Pierce (Rockfold, IL); rabbit polyclonal anti-FGFR4 antibody (C-16) from Santa Cruz Biotechnology, Inc. (Santa Cruz, CA); mouse monoclonal
Table I. Expression of FGFR4 and background in PanIN lesions.

\begin{tabular}{lllll}
\hline & & \multicolumn{2}{c}{ Expression of FGFR4 } \\
\cline { 3 - 4 } Case Age Sex Diagnosis & $\begin{array}{c}\text { Low-grade } \\
\text { PanIN }(\mathrm{n}=10)\end{array}$ & $\begin{array}{c}\text { High-grade } \\
\text { PanIN (n=11) }\end{array}$ \\
\hline
\end{tabular}

\begin{tabular}{rrllll}
\hline 1 & 70 & M & PDAC & - & + \\
2 & 71 & M & PDAC & N & + \\
3 & 61 & M & IPMN & - & + \\
4 & 57 & M & IPMN & N & + \\
5 & 84 & M & CBDC & - & N \\
6 & 75 & F & IPMN & N & + \\
7 & 74 & F & IPMN & - & - \\
8 & 73 & F & IPMN & - & N \\
9 & 68 & F & IPMN & - & + \\
10 & 74 & F & PDAC & - & + \\
11 & 74 & F & IPMN & + & + \\
12 & 87 & M & PDAC & - & + \\
13 & 66 & M & Vater & - & +
\end{tabular}

$1 / 10(10.0 \%) \quad 10 / 11(90.9 \%)^{\mathrm{a}}$

${ }^{\mathrm{a}} \mathrm{P}=0.0003$. M, male; F, female; PDAC, pancreatic ductal adenocarcinoma; IPMN, intraductal papillary mucinous neoplasm; CBDC, common bile duct carcinoma; Vater, carcinoma of Vater's papilla; $\mathrm{N}$, not including the lesion of the sample.

anti-Ki-67 antibody (M7240) from Dako Japan (Tokyo, Japan); HRP-labeled goat anti-rabbit IgG antibody (A102PU) from American Qualex International, Inc. (San Clemente, CA); 35-mm glass-bottom dish from Matsunami Glass Ind., Ltd. (Osaka, Japan); recombinant human FGF19 (rhFGF19) and bovine fibronectin from R\&D Systems, Inc. (Westerville, $\mathrm{OH})$; Histofine Simple Stain Max PO (R) or (M) kit from Nichirei Biosciences, Inc. (Tokyo, Japan); Alexa 488-labeled donkey anti-mouse IgG antibody from Invitrogen Corporation (Carlsbad, CA); Vectashield H-1200 containing 4',6diamidino-2-phenylindole-2HCl (DAPI) from Vector Lab., Inc. (Burlingame, CA); WST-8 cell counting kit from Wako Pure Chemical Industries (Osaka, Japan); $8.0-\mu \mathrm{m}$ pore size cell culture insert and BioCoat Matrigel invasion chamber from Becton-Dickinson, and Company (Franklin Lakes, NJ); Diff-Quick staining kit from Sysmex International Reagents Co., Ltd. (Hyogo, Japan); bovine dermis acid-solubilized type I collagen from KOKEN Co., Ltd. (Tokyo, Japan); Cancer Pathway Finder RT ${ }^{2}$ Profiler PCR Array, RT $^{2}$ First strand kit and $\mathrm{RT}^{2}$ real-time PCR SYBR-Green/ROX from SABiosciences Corporation (Frederick, MD). All other chemicals and reagents were purchased from Sigma-Aldrich Corporation (St. Louis, MO).

Patients and tissues. Pancreatic tissues of PanIN lesions were obtained from thirteen patients (mean age 71.8 years, Table I), who were treated at Nippon Medical School Hospital (Tokyo, Japan) from 2007 to 2009. They required surgery owing to PDAC, intraductal papillary mucinous neoplasm 
Table II. Immunohistochemical analysis of FGFR4 in PDAC cases and clinicopathological factors.

\begin{tabular}{|c|c|c|c|}
\hline & Expression & of FGFR4 & \\
\hline & $\begin{array}{c}\text { Positive } \\
(>30 \%, \mathrm{n}=39)\end{array}$ & $\begin{array}{c}\text { Negative } \\
(\leq 30 \%, n=14)\end{array}$ & P-value \\
\hline Age & & & \\
\hline 63.11 & & & \\
\hline$<65$ & 19 & 3 & 0.1149 \\
\hline$\geq 65$ & 20 & 11 & \\
\hline Sex & & & \\
\hline $\mathrm{F}$ & 16 & 4 & 0.5274 \\
\hline M & 23 & 10 & \\
\hline UICC cl & & & \\
\hline T-prima & & & \\
\hline 1 & 2 & 1 & 0.5433 \\
\hline 2 & 2 & 0 & \\
\hline 3 & 10 & 6 & \\
\hline 4 & 25 & 7 & \\
\hline $\begin{array}{l}\text { N-lymp } \\
\text { metastas }\end{array}$ & & & \\
\hline No & 12 & 6 & 0.5147 \\
\hline $\mathrm{N} 1-3$ & 27 & 8 & \\
\hline $\begin{array}{l}\text { M-dista } \\
\text { metasta }\end{array}$ & & & \\
\hline 0 & 38 & 13 & 0.4623 \\
\hline 1 & 1 & 1 & \\
\hline $\begin{array}{l}\text { G-histol } \\
\text { grading }\end{array}$ & & & \\
\hline G1 & 22 & 6 & 0.8794 \\
\hline G2 & 14 & 7 & \\
\hline G3 & 3 & 1 & \\
\hline Stage & & & \\
\hline I & 2 & 1 & 0.4372 \\
\hline II & 1 & 0 & \\
\hline III & 8 & 3 & \\
\hline IV & 28 & 10 & \\
\hline
\end{tabular}

UICC, International Union Against Cancer.

(IPMN), common bile duct carcinoma (CBDC), or carcinoma of Vater's papilla, and PanIN lesions that were located separately from cancer lesions were obtained for this study. None of the patients received preoperative chemotherapy or radiotherapy. These lesions were classified as low-grade PanIN (including PanIN 1A and 1B) and high-grade PanIN (including PanIN 2 and 3) in terms of both morphology [hematoxylin and eosin (H\&E) staining] and immunohistochemistry (anti-Ki-67) by two pathologists in a blinded manner, following the WHO classification (3). We obtained
10 lesions of low-grade PanIN and 11 lesions of high-grade PanIN, with an overlap of the two types in one case (Table I).

Pancreatic tissues from 53 patients with invasive PDAC were obtained for this study. These patients were treated at Nippon Medical School Hospital from 1995 to 2005. None of these patients received preoperative chemotherapy or radiotherapy. The patients consisted of 33 men and 20 women, whose mean age was 63.7 years (range, 35-84 years). The clinicopathological stage was determined according to the TNM classification system of the International Union Against Cancer and additionally characterized according to the Japan Pancreas Society classification (Table II). Twenty-three patients did not receive any postoperative chemotherapy, whereas 30 patients received adjuvant chemotherapy after surgery. Fourteen patients received Uracil/Tegafur, and 15 patients received gemcitabine. The median follow-up period was 14.7 months. Paraffin-embedded specimens were prepared for immunohistochemical analysis as described previously (21). This study was carried out in accordance with the principles embodied in the Declaration of Helsinki, 2008, and informed consent for the use of pancreatic tissues was obtained from each patient. Normal pancreatic tissue (HuFPT079a) gathered from a 21-year-old woman's pancreatic tail was purchased from US Biomax, Inc. (Rockville, MD).

Pancreatic cancer cell lines. PANC-1, MIA PaCa-2, KLM-1, PK-1 and PK-8 PDAC cell lines were obtained from the Cell Resource Center for Biomedical Research, Institute of Development, Aging and Cancer, Tohoku University (Sendai, Japan), and Capan-1 was purchased from American Type Culture Collection (Manassas, VA). The cells were grown in RPMI-1640 medium containing 10\% fetal bovine serum (FBS), $200 \mathrm{U} / \mathrm{ml}$ penicillin, and $200 \mu \mathrm{g} / \mathrm{ml}$ kanamycin at $37^{\circ} \mathrm{C}$ under a humidified $5 \%$ carbon dioxide atmosphere. Capan-1 was incubated in the same medium with $15 \%$ FBS.

Immunohistochemistry and immunofluorescence. Paraffinembedded sections $(3 \mu \mathrm{m})$ of all tissues described above were subjected to immunostaining using a Histofine Simple Stain MAX PO (R) kit for rabbit polyclonal anti-human FGFR4 antibody (1:100 dilution). In addition, serial sections of PanIN tissues were subjected to H\&E staining and immunostaining using a Histofine Simple Stain MAX PO (M) kit for mouse monoclonal anti-human Ki-67 antibody (1:100 dilution) and five representative cases each of FGFR4positive and -negative PDAC cases were also immunostained for anti-human Ki-67. After deparaffinization, the sections were pretreated in an autoclave at $121^{\circ} \mathrm{C}$ for $10 \mathrm{~min}$ in $10 \mathrm{mmol} / \mathrm{l}$ citrate buffer ( $\mathrm{pH}$ 6.0) for anti-FGFR4 and anti$\mathrm{Ki}-67$. Then, the endogenous peroxidase activity was blocked by incubation for $30 \mathrm{~min}$ with $0.3 \%$ hydrogen peroxide in methanol. The tissue sections were incubated with the primary antibodies in phosphate-buffered saline (PBS) containing $1 \%$ bovine serum albumin (BSA) for $16 \mathrm{~h}$ at $4^{\circ} \mathrm{C}$. Bound antibodies were detected with the Simple Stain MAX PO (R) reagent for anti-FGFR4 antibody and the Simple Stain MAX PO (M) reagent for anti-Ki-67 antibody, using diaminobenzidine tetrahydrochloride as the substrate. The sections were then counterstained with Mayer's hematoxylin. 
Immunohistochemical results for FGFR4 were evaluated as follows: two investigators separately evaluated all the specimens in a blinded manner. The intensity of FGFR4 immunoreactivity of tumor cytoplasm and/or membrane was classified as low or high. When staining was noted in $>30 \%$ of the tumor cells with high intensity on average by two investigators, the case was designated as positive.

For immunofluorescence for anti-FGFR4, cells were cultured on glass-bottom dishes, fixed in $4 \%$ paraformaldehyde/PBS, and permeabilized in $50 \mathrm{mM}$ glycine/PBS for 6 min. After blocking using 10\% goat serum, incubation with primary antibodies for FGFR4 (1:50 dilution) was carried out. After that, detection was carried out by incubation with Alexa Fluor 488-conjugated anti-rabbit IgG (1:1000 dilution) for $1 \mathrm{~h}$ at room temperature and counterstained upon mounting with DAPI. Immunofluorescent images were obtained using a confocal laser microscope (Digital Eclipse C1 TE-2000E, Nikon Instech Co. Ltd., Tokyo, Japan) and analyzed by EZC1 (version 3.8, Nikon Instech Co. Ltd.). The excitation wavelength for FITC (fluorescein-4-isothiocyanate) was $488 \mathrm{~nm}$, and emission was selected and recorded using a 515-530-nm band-pass filter. In addition, the excitation wavelength for DAPI was $405 \mathrm{~nm}$, and emission was selected and recorded using a 435-450-nm band-pass filter.

Quantitative RT-PCR. Total RNA was extracted from PDAC cell lines using FastPure RNA kit according to the manufacturer's protocol. Total RNA sample $(1 \mu \mathrm{g})$ was used for reverse transcription (RT) with the High Capacity cDNA reverse transcription kit following the manufacturer's protocol. Quantitative reverse transcription PCR (qRT-PCR) was performed using a Universal ProbeLibrary System and LightCycler 480 system (Roche Diagnostics). The primer used for FGFR4 corresponded to nts 67-84 (5'-CTG-GAGCTG-GGA-GTG-AGG-3') and nts 121-139 (5'-GCT-GGACTT-CCC-ACC-AAC-T-3') of the human FGFR4 (73 bp, accession no. NM_002011). 18S ribosomal RNA was amplified using the following primer pairs: nts 83-103 (5'-TGCGAG-TAC-TCA-ACA-CCA-ACA-3') and nts 150-167 (5'-GCA-TAT-CTT-CGG-CCC-ACA-3') (85 bp, accession no. NM_022551.2). PCR reaction mixture $(20 \mu \mathrm{l})$ containing $2 \mu \mathrm{l}$ of template cDNA, $0.5 \mu \mathrm{M}$ of each primer, $10 \mu \mathrm{l}$ of $2 \mathrm{x}$ LightCycler 480 Probes Master, and $0.2 \mu \mathrm{M}$ hydrolysis probes as Universal ProbeLibrary no. 72 for FGFR4 or no. 70 for $18 \mathrm{~S}$ rRNA was introduced into a capillary tube. qRT-PCR was performed in a LightCycler 480 and the PCR products were analyzed by LightCycler Data Analysis software version 3.5 (Roche Diagnostics). The optimized program involved denaturation at $95^{\circ} \mathrm{C}$ for 5 min followed by 45 cycles of amplification at $95^{\circ} \mathrm{C}$ for $10 \mathrm{sec}$ and at $60^{\circ} \mathrm{C}$ for $30 \mathrm{sec}$. To confirm amplification specificity, PCR products were subjected to a melting-curve analysis. Results are expressed as FGFR4/ $18 \mathrm{~S}$ rRNA as an internal standard concentration ratio. Gene expression measurements were performed in triplicate.

Western blot analysis. Protein was extracted from cells in accordance with the protocol for the use of the M-Per Mammalian Protein Extraction reagent. Lysates were centrifuged for $10 \mathrm{~min}$ at $13,000 \mathrm{rpm}$ to pellet cell debris and the resulting supernatants were collected. The concentration of protein was measured by the Bradford method. The cleared protein lysates from the cancer cells were subjected to sodium dodecyl sulfate-polyacrylamide gel electrophoresis under reducing conditions, and the separated proteins were transferred to Immobilon P transfer membranes. The membranes were immersed in 5\% skim milk in Tris-buffered saline containing $0.05 \%$ Tween-20 for $60 \mathrm{~min}$ at room temperature, then incubated for $16 \mathrm{~h}$ at $4^{\circ} \mathrm{C}$ with an anti-FGFR 4 antibody (1:500 dilution, the same antibody as for immunohistochemistry). The membranes were washed and incubated with the HRP-conjugated anti-rabbit IgG antibody (1:4000 dilution) for $60 \mathrm{~min}$. After washing, the blots were visualized using enhanced chemiluminescence and detected with a ChemiDoc XRS system (Bio-Rad Laboratories, Inc., Hercules, CA). To confirm the nearly equal loadings of the proteins from the cancer cells, the membranes were washed and reblotted with an anti- $\beta$-actin antibody.

Morphological analysis under the treatment of rhFGF19. In order to elucidate the effect of FGF19 on cell morphology, we incubated MIA PaCa-2 and PANC-1 with or without rhFGF19 (100 ng/ml) in RPMI-1640 medium containing $1 \mu \mathrm{g} / \mathrm{ml}$ heparin. After $24 \mathrm{~h}$, the cells with a subconfluent condition were photographed using a phase-contrast microscope at x100 magnification (Nikon Eclipse TE2000-U, Nikon Instech Co. Ltd.).

Cell proliferation assay under the treatment of rhFGF19. We used WST-8 assay to examine the influence of FGF19 on cell proliferation in vitro. MIA PaCa- 2 and PANC-1 were plated at a density of $5 \times 10^{3}$ in triplicate in 96 -well plates with $0.1 \%$ FBS-containing medium, heparin $(1 \mu \mathrm{g} / \mathrm{ml})$ and rhFGF19 $(0$, 10,50 and $100 \mathrm{ng} / \mathrm{ml}$ ) were added, and samples were incubated at $37^{\circ} \mathrm{C}$ in a humidified $5 \% \mathrm{CO}_{2}$ atmosphere. After $24,48,72$ and $96 \mathrm{~h}, 10 \mu \mathrm{l}$ of WST-8 solution was added to each well, and the cells were incubated at $37^{\circ} \mathrm{C}$. After incubation for $4 \mathrm{~h}$, absorbance was measured at $450 \mathrm{~nm}$. To confirm cell proliferation, we also performed Ki-67 immunostaining with or without rhFGF19 administration, and analyzed Ki-67 labeling index.

Boyden chamber assay under the treatment of rhFGF19. To investigate vertical migration ability under the treatment of rhFGF19 in pancreatic cancer cells, we used the Boyden chamber assay. Uncoated inserts $(8-\mu \mathrm{m}$ pore size, $6.5-\mathrm{mm}$ diameter inserts) were used. FBS $10 \%(750 \mu 1)$ containing RPMI-1640 medium was added to the bottom of each well and MIA PaCa- 2 were seeded at $1.0 \times 10^{5}$ in the inserts. Before incubation, heparin and rhFGF19 were administered at $1 \mu \mathrm{g} / \mathrm{ml}$ and 0,10 and $100 \mathrm{ng} / \mathrm{ml}$. After incubation for $16 \mathrm{~h}$ (MIA PaCa-2) at $37^{\circ} \mathrm{C}$ in a humidified $5 \% \mathrm{CO}_{2}$ atmosphere, the non-migrated cells were swept away from the upper surface of the membrane. Migrated cells were stained using a Diff-Quick stain kit following the manual of the manufacturer. After staining, the migrated cells were counted in five fields of each membrane at x200 magnification. Boyden chamber assays were performed in triplicate.

Time-lapse analysis under the treatment of rhFGF19. To investigate linear migration ability under the treatment of 
rhFGF19 in pancreatic cancer cells, we observed cell migration with or without rhFGF19 by time-lapse photography. MIA PaCa-2 were seeded at $1.0 \times 10^{4}$ per well in a $35-\mathrm{mm}$ glass-bottom dish. After incubation for $48 \mathrm{~h}$, these media were removed and $0.1 \%$ FBS and $1 \mu \mathrm{g} / \mathrm{ml}$ heparin with RPMI-1640 medium were added with or without rhFGF19 at $100 \mathrm{ng} / \mathrm{ml}$. After the administration, these cells were measured at intervals of $5 \mathrm{~min}$ for $24 \mathrm{~h}$ using a phase contrast microscope (Digital Eclipse C-1 TE-2000E, Nikon Instech Co. Ltd.). The migration distance in each dish was measured and analyzed by MetaMorph software (Universal Image Corporation Ltd., Buckinghamshire, UK). Time-lapse analysis was performed on 20 cells for each condition.

Matrigel invasion assay under the treatment of rhFGF19. We carried out invasion assay using BD BioCoat Matrigel Invasion Chamber to determine the effects on invasion ability of the absence or presence of rhFGF19. Before use, medium was added to inserts and wells, which were incubated for $2 \mathrm{~h}$ at $37^{\circ} \mathrm{C}$ in a humidified $5 \% \mathrm{CO}_{2}$ atmosphere, and removed carefully. MIA PaCa- 2 were plated at $1.0 \times 10^{5}$ in each insert and $750 \mu 1$ of $10 \%$ FBS-containing RPMI-1640 medium was added to the bottom of each well. Before incubation, $1 \mu \mathrm{g} / \mathrm{ml}$ heparin and 0 or $100 \mathrm{ng} / \mathrm{ml} \mathrm{rhFGF} 19$ were added. Staining and cell counting were carried out as mentioned above for the Boyden chamber assay.

Cell adhesion to extracellular matrix under the treatment of rhFGF19. Bovine type I collagen, human type IV collagen, bovine fibronectin and murine laminin solutions at a concentration of $20 \mu \mathrm{g} / \mathrm{ml}$ were added to the wells of 96-well microplates followed by incubation at $37^{\circ} \mathrm{C}$ in a humidified $5 \% \mathrm{CO}_{2}$ atmosphere overnight. The residual culture area was blocked with $0.3 \% \mathrm{BSA}$ in PBS at $37^{\circ} \mathrm{C}$ in a humidified $5 \% \mathrm{CO}_{2}$ atmosphere for $2 \mathrm{~h}$. Cell suspension $(100 \mu \mathrm{l})$ of MIA PaCa-2 was seeded at $2 \times 10^{4}$ cells/well and heparin $(1 \mu \mathrm{g} / \mathrm{ml})$ was added with or without $\mathrm{rhFGF} 19(100 \mathrm{ng} / \mathrm{ml})$; then, samples were incubated for $4 \mathrm{~h}$ at $37^{\circ} \mathrm{C}$ in a humidified $5 \% \mathrm{CO}_{2}$ atmosphere. Non-adherent cells were removed by washing with serum-free medium. The relative number of attached cells was determined using a WST-8 cell counting kit. All assays were performed in triplicate.

PCR array analysis. We incubated MIA PaCa- 2 with heparin $(1 \mu \mathrm{g} / \mathrm{ml})$ in the absence or presence of $\operatorname{rhFGF} 19(100 \mathrm{ng} / \mathrm{ml})$. As previously described, total RNA was extracted from MIA PaCa-2 with or without rhFGF19 $24 \mathrm{~h}$ after administration. For PCR array experiments, $\mathrm{RT}^{2}$ Profiler PCR array was used to simultaneously examine the mRNA levels of 89 genes closely associated with cancer progression, including five house-keeping (HK) genes in 96-well plates following the manufacturer's protocol. Briefly, first-strand cDNAs were synthesized from $1 \mu \mathrm{g}$ of total RNA using $\mathrm{RT}^{2}$ First-strand kit, and then mixed with $\mathrm{RT}^{2}$ real-time PCR SYBR-Green/ ROX according to the manufacturer's protocol. qRT-PCR was carried out in an ABI-7900HT Standard 96 Block (Applied Biosystems, Inc). Cycling conditions were as follows: $10 \mathrm{~min}$ at $95^{\circ} \mathrm{C}$ and then 40 cycles of amplification $\left(95^{\circ} \mathrm{C}\right.$ for $15 \mathrm{sec}$, $60^{\circ} \mathrm{C}$ for $1 \mathrm{~min}$ ). To confirm amplification specificity, PCR products were subjected to a melting-curve analysis. Values were obtained for the threshold cycle $(C t)$ for each gene and normalized using the average of five HK genes on the same array (B2M, HPRT1, RPL13A, GAPDH, and ACTB). Ct values for HK genes were monitored for consistency between the arrays. Changes between cells with or without rhFGF19 addition were determined by the $\Delta \Delta C t$ method: $\Delta C t=C t$ $C t_{(H K)}, \Delta \Delta C t=C t_{(F G F 19+)}-C t_{(F G F 19-)}$, and the resulting values were reported as fold change $=2^{(-\Delta \Delta C t)}$. Negative controls ensured the absence of DNA contamination and set the threshold for absence/presence.

Statistical analysis. All quantitative data are presented as mean \pm SE values, and Chi-square test and Fisher's exact test were used to analyze the correlation between FGFR4 expression and clinicopathological features. Fisher's exact test was used to analyze the relationship between FGFR4 expression and PanIN grading.

Cumulative survival rate was calculated by the KaplanMeier method, and the significance of differences in survival rate was analyzed by the log-rank test. Results of the cultured cells were assessed using Student's t-test. $\mathrm{P}<0.05$ was considered significant in all analyses. Computations were performed using GraphPad Prism 5 software package (GraphPad software, La Jolla, CA).

\section{Results}

Immunohistochemical analysis of FGFR4 in PanIN tissues. We analyzed the expression of FGFR4 in both low-grade PanIN (10 lesions) and high-grade PanIN (11 lesions) immunohistochemically. Representative cases of PanIN are shown in Fig. 1. FGFR4 was not detected in low-grade PanIN lesions (Fig. 1B), in which the proportion of Ki-67-positive cells was extremely low (Fig. 1C). In contrast, FGFR4 was highly expressed in high-grade PanIN lesions (Fig. 1E), in which the proportion of Ki-67-positive cells was high (Fig. 1F). There was a significant difference in FGFR4 expression between low-grade and high-grade PanIN lesions ( $\mathrm{P}=0.0003)$. FGFR4 was expressed in only $10.0 \%$ of low-grade PanIN, while its expression was detected in $90.9 \%$ of high-grade PanIN lesions (Table I). The immunostaining of FGFR4 could clarify the transition from low-grade to high-grade PanIN (Fig. 1G and $\mathrm{H}$ ) from the difference in the distribution of Ki-67positive cells (Fig. 1I).

Immunohistochemical analysis of FGFR4 in PDAC tissues and clinicopathological analysis. Immunohistochemical analysis of the PDAC samples was performed next to determine whether there was a correlation between FGFR4 expression in the cancer cells and clinicopathological features. FGFR4 was localized in the cytoplasm and cell membrane of the cancer cells in 39 of the 53 cases (73.6\%) (Fig. 2A and B). Clinicopathological factors were not correlated with the expression of FGFR4 (Table II). We also analyzed the relation between FGFR4 expression and proliferation; however, Ki-67 labeling index did not differ between FGFR4-positive and -negative PDAC cases (data not shown). In normal pancreatic tissue, FGFR4 was only expressed in islet cells (Fig. 2C), but was not detected in normal duct (Fig. 2D), whereas mesenchymal cells were negative for FGFR4. 

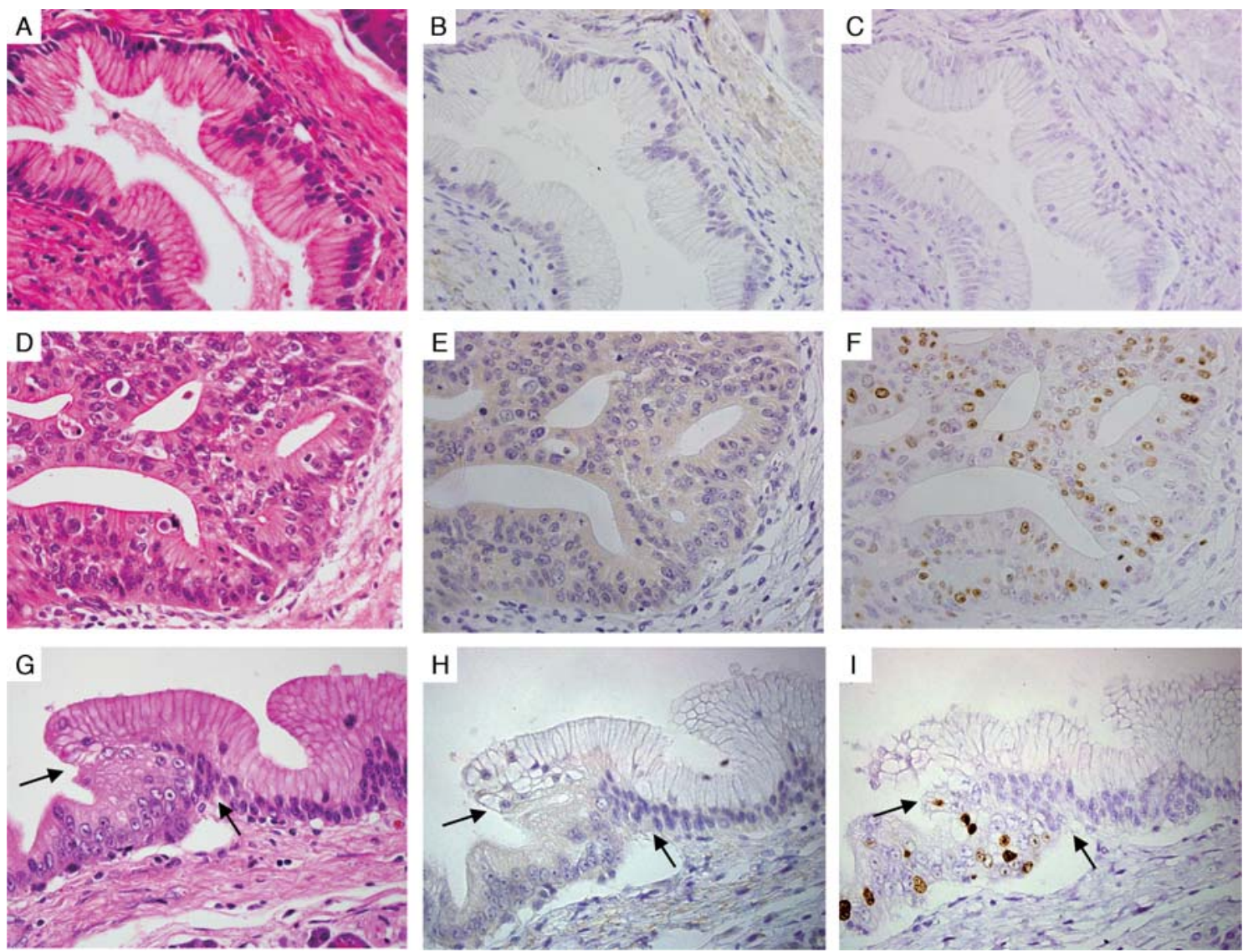

Figure 1. Immunohistochemical analysis of FGFR4 in PanIN lesions. (A, B and C) Typical low-grade PanIN lesion (PanIN-1A). FGFR4 was not expressed (B) and the frequency of Ki-67-positive cells was extremely low (C). (D, E and F) Typical high-grade PanIN lesion (PanIN-3). FGFR4 was diffusely expressed in neoplastic cells (E) and the frequency of Ki-67-positive cells was high (F). (G, H and I) Transition from low-grade to high-grade PanIN (arrows), which was evident in the difference in the number of Ki-67-positive cells (I). The expression of FGFR4 was only detected in high-grade PanIN (H). Panels (A, D and G) hematoxylin and eosin staining. (B, E and H) Anti-FGFR4 immunostaining. (C, F and I) Anti-Ki-67 immunostaining. Original magnification, $\mathrm{x} 600$.
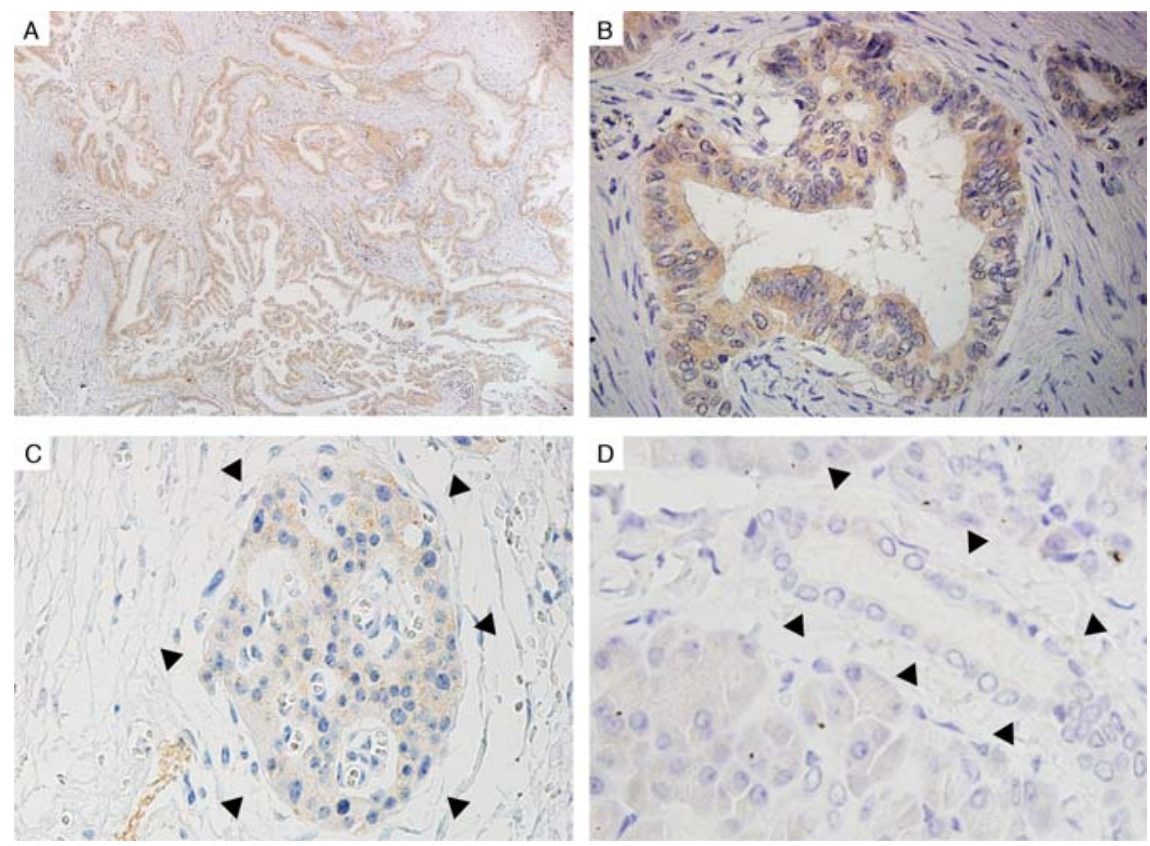

Figure 2. Immunohistochemical analysis of FGFR4 in pancreatic cancer and normal pancreas. (A and B) Characteristic staining patterns of FGFR4 in invasive PDAC cases. FGFR4 immunoreactivity was detected in the cytoplasm and cell membrane of PDAC cells. (C and D) FGFR4 was expressed in islet tissue (C, arrowheads), but was not expressed in normal duct (D, arrowheads). Original magnification: panel A, x100; B and D, x600; C, x400. 


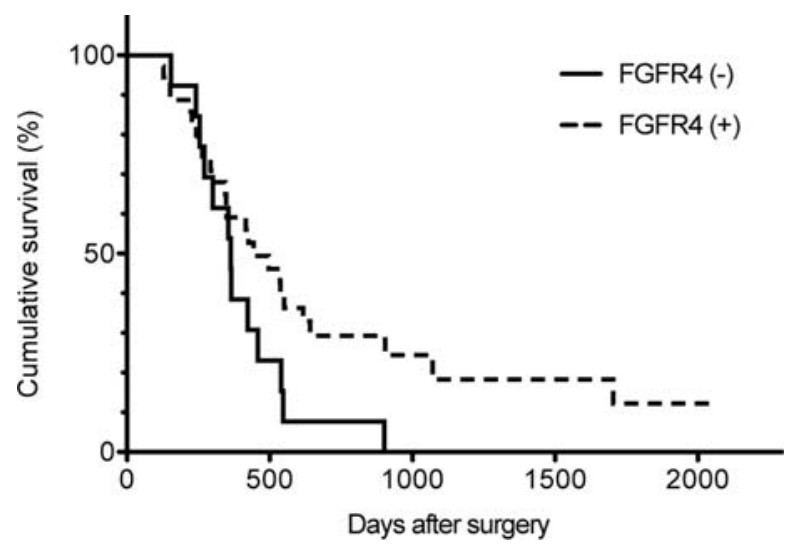

Figure 3. Cumulative Kaplan-Meier survival curve. (A) Curves for patients with FGFR4-positive and -negative tumors. The survival of the FGFR4positive group tended to be longer than that of the FGFR4-negative group, but the difference was not significant $(\mathrm{P}=0.068)$.
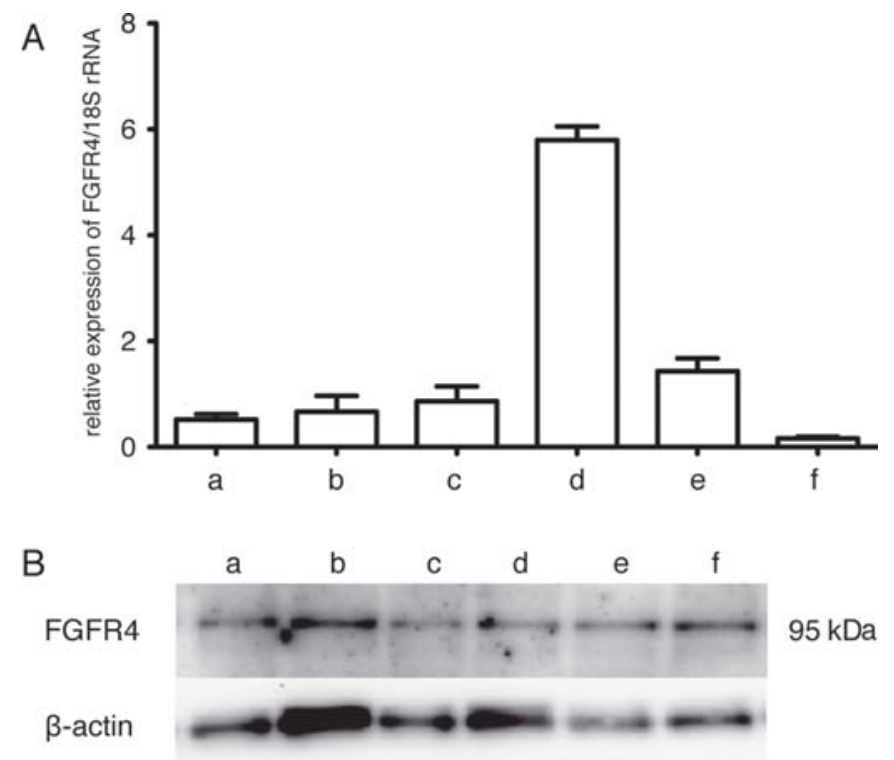

C

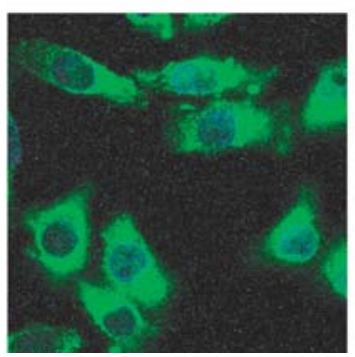

D

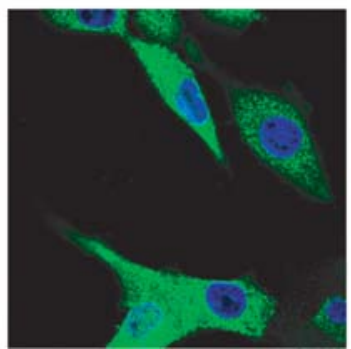

Figure 4. FGFR4 expression in PDAC cell lines. (A) qRT-PCR analysis of FGFR4 in six PDAC cell lines: a, PANC-1; b, MIA PaCa-2; c, KLM-1; d, Capan-1; e, PK-1; and f, PK-8. FGFR4 was expressed in all six cell lines. The highest and lowest mRNA levels of FGFR4 were observed in Capan-1 and PK-8, with 5.80 and 0.16 times the mRNA level of 18S RNA, respectively. The mRNA expression was measured in triplicate and is shown as the mean \pm SE. (B) Western blot analysis of FGFR4 in the same six PDAC cell lines as above. A band corresponding to the $95-\mathrm{kD}$ FGFR4 protein was detected in all pancreatic cancer cell lines (upper panel). ß-actin served as a loading control (lower panel). (C and D) Immunofluorescent analysis of FGFR4 in MIA PaCa-2 (C) and PANC-1 (D). FGFR4 was detected in the cell membrane and/or cytoplasm in both cells (green). The nuclei were counterstained by DAPI (blue). (C and D) Original magnification x1000.

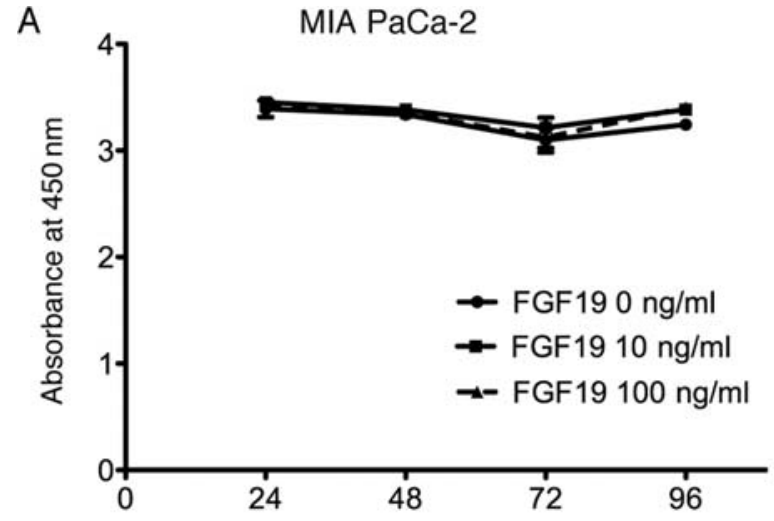

Time after administration of FGF19 (h)

B

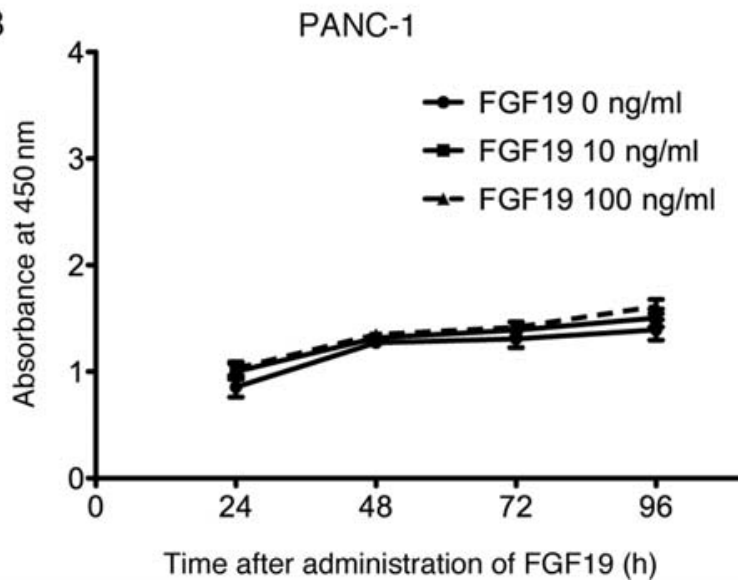

Figure 5. Cell proliferation assay with or without rhFGF19 administration in MIA PaCa-2 (A) and PANC-1 (B). (A and B) These cells were plated at a density of $5 \times 10^{3}$ in 96-well plates, and then the cells were incubated for 24 , 48, 72 and $96 \mathrm{~h}$ after administration of rhFGF19 (at 0,10 , or $100 \mathrm{ng} / \mathrm{ml}$ ). FGF19 did not significantly affect the cell proliferation in either cell line.

Cumulative Kaplan-Meier survival curve of PDAC patients. The overall 2-year survival rate of all 53 cases of PDAC was $15.1 \%$. The overall survival of the FGFR4-positive group tended to be longer than that of the FGFR4-negative group, but the difference was not significant $(\mathrm{P}=0.068)$ (Fig. 3).

FGFR4 expression in pancreatic cancer cell lines. qRT-PCR analysis was performed to determine the expression level of FGFR4 mRNA in 6 PDAC cell lines: PANC-1, MIA PaCa-2, KLM-1, Capan-1, PK-1 and PK-8. All 6 cell lines expressed FGFR4, at variable levels (Fig. 4A). The FGFR4 mRNA level was the highest in Capan-1 cells and the lowest in PK-8 cells.

Western blot analysis was carried out to assess the expression of FGFR 4 protein in the same 6 PDAC cell lines as for qRT-PCR analysis. A $95-\mathrm{kD}$ band corresponding to FGFR4 and a $42-\mathrm{kD}$ band corresponding to $\mathrm{B}$-actin were detected in all cell lines (Fig. 4B upper and lower panels, respectively).

To examine the intracellular localization of FGFR4 protein in PDAC cells, immunofluorescent analysis using confocal laser microscopy was performed in PANC-1 and MIA PaCa-2. FGFR4 protein was expressed in the cytoplasm and the cell membrane in both cell lines (Fig. 4C and D).

Effects on cell proliferation and cell morphology of rhFGF 19 stimulation. To clarify the role of FGFR4 in PDAC, the 

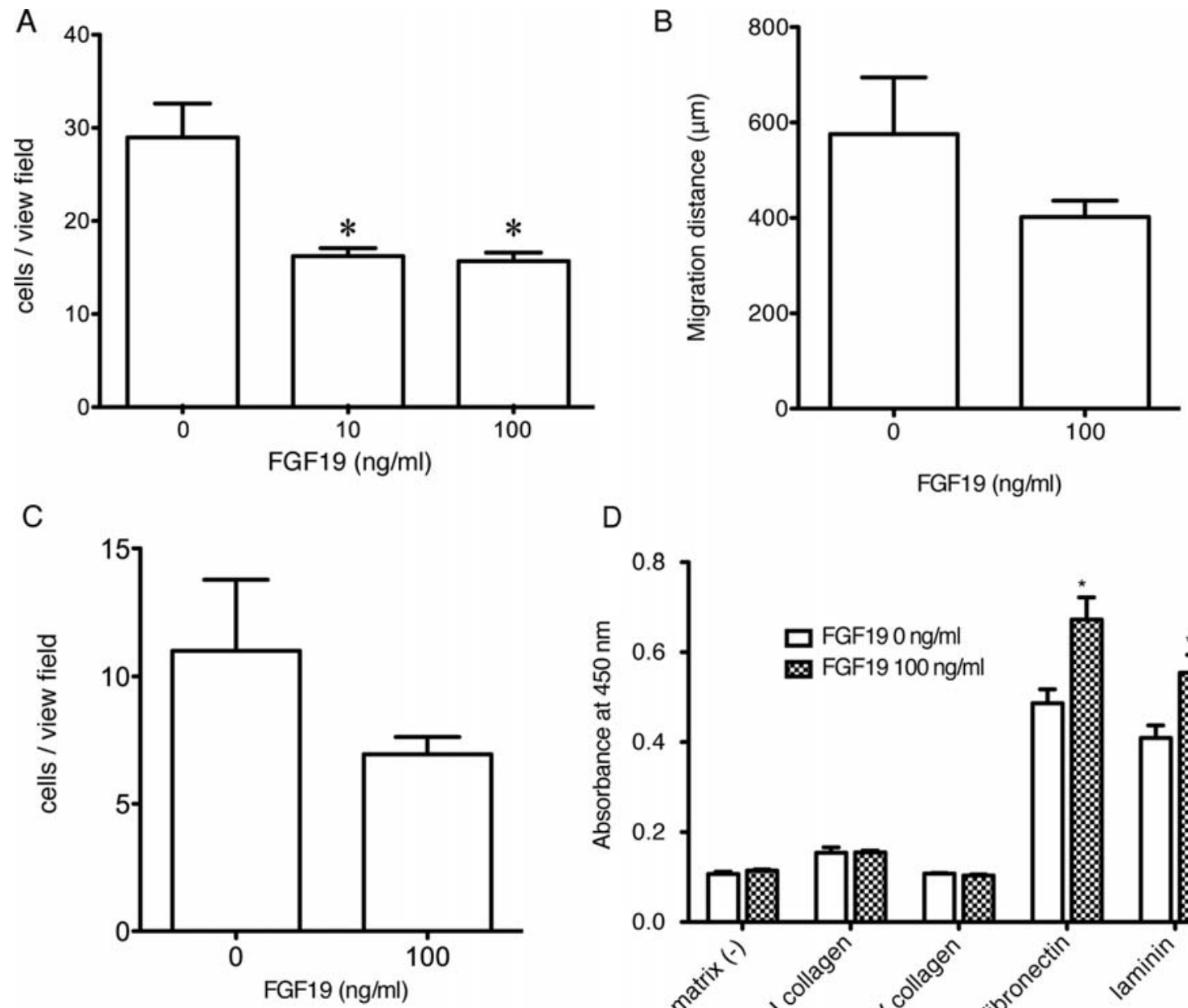

D

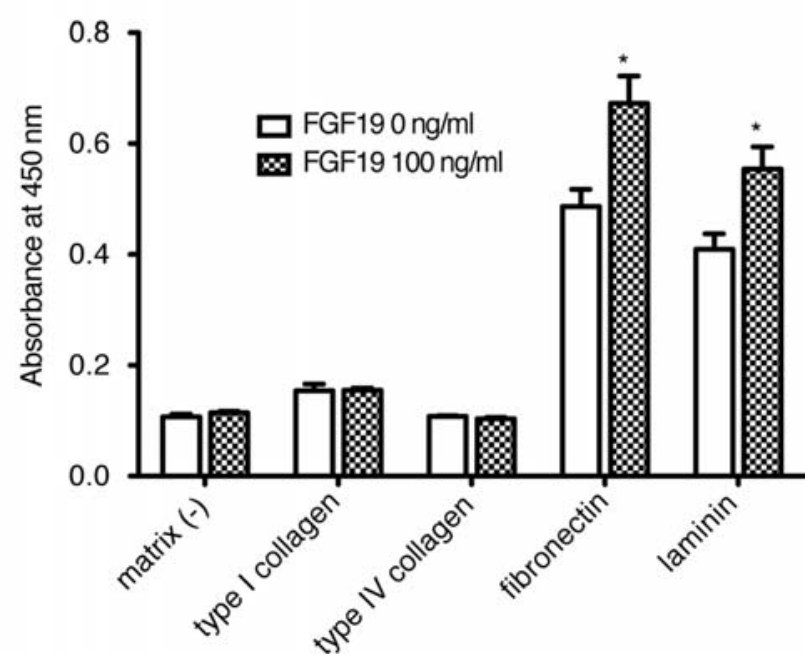

Figure 6. Effects of FGF19 for cell migration (A and B), invasion (C), and attachment assay (D) in MIA PaCa-2. (A) Boyden chamber assay with or without the administration of rhFGF19. The migrated cell counts per view field were significantly decreased in the FGF19-treated cells (at 10 and $100 \mathrm{ng} / \mathrm{ml}$ ) compared with those of the untreated cells $\left({ }^{*} \mathrm{P}<0.05\right)$. (B) Time-lapse analysis for $24 \mathrm{~h}$ after rhFGF19 administration in MIA PaCa-2. Migrated cells were photographed every $5 \mathrm{~min}$ and the migrated distance was measured using MetaMorph for 20 cells arbitrarily selected in each condition. Although there was no significant difference between treated and untreated cells, FGF19-administered cells also tended to be inhibited in terms of horizontal migration. (C) The administration of rhFGF19 had no significant effect on cell invasion, but rhFGF19 had a tendency to inhibit cell invasion in MIA PaCa-2. (D) Cells that adhered to ECMs were measured using a WST-8 kit and described in terms of absorption at $450 \mathrm{~nm}$ after $2 \mathrm{~h}$ of FGF19 administration. The administration of rhFGF19 significantly increased the number of cells adherent to fibronectin and laminin in MIA PaCa-2 $\left({ }^{*} \mathrm{P}<0.05\right)$.

administration of rhFGF19 was examined and found not to influence the cell morphology in MIA PaCa-2 and PANC-1 (data not shown). We investigated PDAC cell lines, MIA PaCa-2 and PANC-1, by the administration of FGFR4-specific ligand, FGF19, for cell proliferation. Exogenous rhFGF19 administration did not affect the cell proliferation in either cell independently of the concentration of rhFGF19 (Fig. 5A and B). Ki-67 labeling index also did not differ, regardless of whether rhFGF19 was administered to MIA PaCa-2 cells (data not shown).

Effects on cell migration, invasion and attachment of rhFGF19 stimulation. We next investigated the effects on cell migration, invasion and attachment of rhFGF19 administration by using MIA PaCa-2. With regard to vertical migration by Boyden chamber assay, MIA PaCa-2 showed a significant decrease in migrated cell counts in the presence of rhFGF19 compared with that in the absence of rhFGF19 (Fig. 6A). Time-lapse analysis revealed that the rhFGF19 also tended to reduce the horizontal migration in MIA $\mathrm{PaCa}-2$ but this effect was not significant (Fig. 6B).

In addition, we assessed cell invasiveness with or without rhFGF19 administration using Matrigel Invasion Chamber, which resembles the basement membrane matrix. Although there was no statistical difference between the two conditions, similar to the result of the Boyden chamber assay, FGF19 tended to reduce cell invasion in MIA PaCa-2 (Fig. 6C).

We further examined the effects on cell attachment to ECM components: type I and IV collagen, laminin and fibronectin. The results showed that the numbers of cells that adhered to laminin and fibronectin were significantly increased in MIA $\mathrm{PaCa}-2$ in the presence of rhFGF19 compared with those in the absence of rhFGF19, but cell adhesion to collagen did not differ by administration of rhFGF19 (Fig. 6D).

Comprehensive cellular pathway analysis of FGF19/FGFR4. To elucidate the mechanism of FGF19/FGFR4 signaling with regard to cell adhesion, we evaluated mRNA levels of selected 
Table III. Summary of genes identified as different in FGF19-treated MIA PaCa2 cells using RT2 Profiler PCR arrays.

\begin{tabular}{|c|c|c|c|c|}
\hline Refseq & Symbol & Description & Function & Fold change \\
\hline NM_000885 & ITGA4 & Integrin $\alpha 4$ (antigen CD49D, $\alpha 4$ subunit of VLA-4 receptor) & Cell adhesion & 6.7173 \\
\hline NM_005163 & AKT1 & V-akt murine thymoma viral oncogene homolog 1 & Cell growth & 6.0915 \\
\hline NM_000594 & TNF & Tumor necrosis factor (TNF superfamily, member 2) & $\begin{array}{l}\text { Cell apoptosis, prolifera- } \\
\text { tion and differentiation }\end{array}$ & 2.6758 \\
\hline NM_003246 & THBS1 & Thrombospondin 1 & Angiogenesis & 2.4763 \\
\hline NM_002421 & MMP1 & Matrix metallopeptidase 1 (interstitial collagenase) & Degradation of ECM & -2.0563 \\
\hline NM_004739 & MTA2 & Metastasis associated 1 family, member 2 & Tumor metastasis & -2.1055 \\
\hline NM_000245 & MET & Met proto-oncogene (hepatocyte growth factor receptor) & $\begin{array}{l}\text { Cell growth, angiogenesis } \\
\text { and metastasis }\end{array}$ & -2.2225 \\
\hline NM_002203 & ITGA2 & Integrin $\alpha 2$ (CD49B, $\alpha 2$ subunit of VLA-2 receptor) & Cell adhesion & -2.3008 \\
\hline NM_004448 & ERBB2 & $\begin{array}{l}\text { V-erb-b2 erythroblastic leukemia viral oncogene homolog } 2 \text {, } \\
\text { neuro/glioblastoma-derived oncogene homolog (avian) }\end{array}$ & $\begin{array}{l}\text { Cell growth } \\
\text { and differentiation }\end{array}$ & -2.3216 \\
\hline NM_014751 & MTSS1 & Metastasis suppressor 1 & Suppress of metastasis & -2.3222 \\
\hline NM_198253 & TERT & Telomerase reverse transcriptase & Regulation of telomerase & -2.6251 \\
\hline NM_006500 & MCAM & Melanoma cell adhesion molecule & Cell adhesion & -2.7336 \\
\hline
\end{tabular}

Fold change expressed as derived $\Delta \Delta \mathrm{Ct}$ values; positive values represent enhanced expression; negative values represent suppressed expression in FGF19-treated MIA PaCa-2 vs. untreated MIA PaCa-2. Only genes that showed a 2-fold or greater value are summarized.

molecules related to tumor progression. The molecules for which the mRNA level in rhFGF19-treated MIA PaCa-2 cells was 2.0-fold or greater than that of non-treated cells are summarized in Table III. Focusing on cell adhesion, the expression of integrin $\alpha 4$ was markedly increased 6.72-fold in rhFGF19-treated cells compared with that in non-treated cells.

\section{Discussion}

Various FGF and FGFR are expressed in PDAC tissues, and several reports suggest that the FGF/FGFR family participates in the progression and carcinogenesis of pancreatic cancer (22). It was previously found in our laboratory that the overexpression of both FGF7 and FGFR2IIIb in pancreatic cancer correlated with venous invasion, vascular endothelial growth factor (VEGF)-A, matrix metalloproteinase (MMP)-9 expression, and poor prognosis (23). FGFR1, 3, or 4 is also expressed in PDAC cell lines with high frequency (over $70 \%$ ) (24). FGFR4 is expressed in various fetal organs, including skeletal muscles, liver, lung, pancreas, adrenal cortex, kidney and brain (25), but the fgfr 4 gene knockout mouse does not show abnormalities, unlike those of other FGFR families (26). Hughes examined the distribution of FGFR1-4 in human tissue by immunohistochemistry, and he revealed that FGFR4 is expressed in various tissues of adults, such as hepatocyte, bile duct epithelium, islet cell of Langerhans, luminal epithelium of stomach, and media of arteriole and vein (27). Recent studies have revealed that a germline single nucleotide polymorphism (SNP) in FGFR4 at codon 388 , from $\mathrm{G}$ to $\mathrm{A}$, results in a change in the amino acid sequence from glycine to arginine (Arg388 allele), and that this polymorphism is correlated with a poor prognosis for various tumors, such as colon (28), prostate (29), breast (28), head and neck (30), and lung cancer (31). However, the role of FGFR4 itself in carcinogenesis is controversial, but, as mentioned above, the incidence of FGFR4 expression and its role in human cancer are not yet elucidated well.

In the present study, the expression of FGFR4 was barely observed in low-grade PanIN lesions but was frequently detected in cell membrane and cytoplasm of high-grade PanIN lesions, including carcinoma in situ. FGFR4 immunoreactivity was observed in cell membrane and cytoplasm of cancer cells in 39 of 53 PDAC tissues $(73.6 \%)$, whereas it was limited not in ductal cells but in islet cells in normal pancreatic tissue. Here, we assumed that FGFR4 expression might occur at a relatively early phase of the carcinogenesis of PDAC; that is, the transition from low-grade PanIN to high-grade PanIN. It was reported that FGFR4 overexpression was mediated by an intronic enhancer activated by a transcription factor, such as hepatic nuclear factor 1 (HNF-1) (32), but it remains unclear whether the same activation occurs in PanIN lesions. The trend in the FGFR4-positive group of PDAC was for longer overall survival than that in the FGFR4negative group, although clinicopathological factors were not correlated with the expression of FGFR4. FGFR4 expression in PDAC culture cell lines in vitro was also verified by qRTPCR, Western blot, and immunofluorescent analyses. These results indicated that FGFR4 expression in PDAC occurred until carcinoma development in situ. 
FGF19 binds to FGFR4 with a high affinity and can activate downstream signaling in the absence of interaction with BKlotho protein, which is assumed to be because the N-terminal region of FGF19 is responsible for the association of FGFR4 with the existence of heparin, whereas the C-terminal region is for ßKlotho $(17,33)$. Preliminary qRT-PCR analyses of PDAC cell lines and immunohistochemistry of PDAC tissues for FGF19 were carried out and it was confirmed that FGF19 was hardly expressed in either PDAC cell lines or tissues. In this study, we next determined the role of FGFR4 in PDAC cell lines by the stimulation of exogenous rhFGF19 administration. Unlike the findings for other FGFR stimulations previously reported (34), the stimulation of FGFR4 by rhFGF19 administration did not affect cell morphology or proliferation in either of the two PDAC cell lines. In contrast, rhFGF19 administration with heparin significantly suppressed vertical migration and also tended to suppress invasion in PDAC cells compared with those to which only heparin was administered. In addition, FGFR4 stimulation was associated with cancer cell adhesion to several ECM components, such as fibronectin and laminin. Compared with non-treated cells, the expression level of integrin $\alpha 4$ was markedly increased in rhFGF19-treated cells. It was indicated that integrin $\alpha 4$ can bind to fibronectin, vascular endothelial cell adhesion molecule-1 (VCAM-1), and mucosa addressing cell adhesion molecule (MadCAM) (35) and that knockdown of the integrin family of MIA $\mathrm{PaCa}-2$ resulted in increased motility and decreased adhesion to ECMs (36). One feature of PDAC, extensive desmoplasia, was severe, because not only fibroblast and pancreatic satellite cells but also cancer cells produced ECM proteins, such as fibronectin and collagen (37). Although the mechanism by which the stimulation of FGFR 4 increased integrin $\alpha 4$ expression was not clarified, these results indicate that the stimulation of FGFR4 by rhFGF19 administration could inhibit cell migration and invasion by increased cell adhesion to some type of cancer-associated stromal ECM owing to upregulation of the integrin family.

It was indicated that the cellular pathway of FGFR4 differed from those of other FGFRs and that it did not induce mitogenic responses in vitro. In rat myoblast cells transfected with FGFR1, FGFR2, or FGFR4, FGFR4-expressing cells did not induce morphological change and anchor-independent cell growth, unlike other FGFR-expressing cells (38). In HCC cell lines expressing FGFR1 or FGFR4, both FGFR1 and FGFR4 stimulation by FGF1 induced activation of Erk, but FGFR4-expressing cells failed to sustain this activation within $30 \mathrm{~min}$, whereas FGFR1-expressing cells sustained this activation for $>1 \mathrm{~h}$ (13). In addition, FGFR4-expressing HCC cells induced a 1.9-fold increase in apoptosis by FGF1 administration compared with that of cells without FGF1 administration, in contrast to FGFR1-expressing HCC cells that induced a $30 \%$ decrease (13). These findings support the result of this study that FGFR4 stimulation does not affect cell morphology and growth. These differences between FGFR4 and other FGFRs might be due to the differences of FGFR4 transmembrane and intercellular domains from those of other FGFRs, as described in a previous investigation using chimeric receptors (39), but further studies concerning signal transduction are necessary.
In summary, this is the first report to indicate that FGFR4 expression is markedly increased in high-grade PanIN and PDAC compared with that in normal and low-grade PanIN. This change of FGFR4 expression probably occurs in a high frequency during the transition from low-grade PanIN to more aggressive disease. Furthermore, we are the first to report that FGFR4 might play a suppressive role, such as inhibition of cell migration and invasion, rather than a progressive role, such as mitogenic response, in PDAC cells in vitro. FGFR4 expression is a promising marker of neoplastic features in pancreatic ductal tissue and exogenous rhFGF19 administration may be an effective tool for the control of aggressiveness of PDAC.

\section{Acknowledgements}

We express our appreciation to Takenori Fujii, Kiyoshi Teduka, Yoko Kawamoto, Taeko Suzuki and Kiyoko Kawahara (Department of Pathology, Integrative Oncological Pathology, Graduate School of Medicine, Nippon Medical School) for their excellent technical assistance. We also thank Dr Shinichi Tsuchiya (Division of Surgical Pathology, Nippon Medical School Hospital) for preparing tissue blocks.

\section{References}

1. Bardeesy $\mathrm{N}$ and DePinho RA: Pancreatic cancer biology and genetics. Nat Rev Cancer 2: 897-909, 2002.

2. Matsuno S, Egawa S, Fukuyama S, et al: Pancreatic cancer registry in Japan: 20 years of experience. Pancreas 28: 219-230, 2004.

3. Klöppel G, Adler G, Hruban RH, Kern SE, Longnecker DS and Partanen TJ: Ductal adenocarcinoma of the pancreas. In: Tumours of the Digestive System. Vol 2. 3rd edition. Hamilton SR and Aaltonen LA (eds). International Agency for Research on Cancer, Lyon, pp221-230, 2000.

4. Feldmann G, Beaty R, Hruban R and Maitra A: Molecular genetics of pancreatic intraepithelial neoplasia. J Hepatobiliary Pancreat Surg 14: 224-232, 2007.

5. Furukawa T: Molecular targeting therapy for pancreatic cancer: current knowledge and perspectives from bench to bedside. J Gastroenterol 43: 905-911, 2008.

6. Eswarakumar V, Lax I and Schlessinger J: Cellular signaling by fibroblast growth factor receptors. Cytokine Growth Factor Rev 16: 139-149, 2005.

7. Xie M, Holcomb I, Deuel B, et al: FGF-19, a novel fibroblast growth factor with unique specificity for FGFR4. Cytokine 11: 729-735, 1999.

8. Ornitz D, Xu J, Colvin J, et al: Receptor specificity of the fibroblast growth factor family. J Biol Chem 271: 15292-15297, 1996.

9. Ho H, Pok S, Streit S, et al: Fibroblast growth factor receptor 4 regulates proliferation, anti-apoptosis and alpha-fetoprotein secretion during hepatocellular carcinoma progression and represents a potential target for therapeutic intervention. J Hepatol 50: 118-127, 2009.

10. Roidl A, Berger H, Kumar S, Bange J, Knyazev P and Ullrich A: Resistance to chemotherapy is associated with fibroblast growth factor receptor 4 up-regulation. Clin Cancer Res 15: 2058-2066, 2009.

11. Takahashi A, Sasaki H, Kim S, et al: Identification of receptor genes in renal cell carcinoma associated with angiogenesis by differential hybridization technique. Biochem Biophys Res Commun 257: 855-859, 1999.

12. Streit S, Mestel D, Schmidt M, Ullrich A and Berking C: FGFR4 Arg388 allele correlates with tumour thickness and FGFR4 protein expression with survival of melanoma patients. Br J Cancer 94: 1879-1886, 2006.

13. Huang X, Yang C, Jin C, Luo Y, Wang F and McKeehan W: Resident hepatocyte fibroblast growth factor receptor 4 limits hepatocarcinogenesis. Mol Carcinog 48: 553-562, 2009. 
14. Strack A and Myers R: Modulation of metabolic syndrome by fibroblast growth factor 19 (FGF19)? Endocrinology 145: 2591-2593, 2004.

15. Song K, Li T, Owsley E, Strom S and Chiang J: Bile acids activate fibroblast growth factor 19 signaling in human hepatocytes to inhibit cholesterol 7alpha-hydroxylase gene expression. Hepatology 49: 297-305, 2009.

16. Goetz R, Beenken A, Ibrahimi O, et al: Molecular insights into the klotho-dependent, endocrine mode of action of fibroblast growth factor 19 subfamily members. Mol Cell Biol 27: 3417-3428, 2007.

17. Wu X, Ge H, Lemon B, et al: Selective activation of FGFR4 by an FGF19 variant does not improve glucose metabolism in ob/ob mice. Proc Natl Acad Sci USA 106: 14379-14384, 2009

18. Pai R, Dunlap D, Qing J, Mohtashemi I, Hotzel K and French D: Inhibition of fibroblast growth factor 19 reduces tumor growth by modulating beta-catenin signaling. Cancer Res 68: 5086-5095, 2008.

19. Desnoyers L, Pai R, Ferrando R, et al: Targeting FGF19 inhibits tumor growth in colon cancer xenograft and FGF19 transgenic hepatocellular carcinoma models. Oncogene 27: 85-97, 2008.

20. Katoh M: Cross-talk of WNT and FGF signaling pathways at GSK3beta to regulate beta-catenin and SNAIL signaling cascades. Cancer Biol Ther 5: 1059-1064, 2006.

21. Ishiwata T, Friess H, Büchler M, Lopez M and Korc M: Characterization of keratinocyte growth factor and receptor expression in human pancreatic cancer. Am J Pathol 153: 213$222,1998$.

22. Kornmann M, Beger HG and Korc M: Role of fibroblast growth factors and their receptors in pancreatic cancer and chronic pancreatitis. Pancreas 17: 169-175, 1998.

23. Cho K, Ishiwata T, Uchida E, et al: Enhanced expression of keratinocyte growth factor and its receptor correlates with venous invasion in pancreatic cancer. Am J Pathol 170: 19641974, 2007.

24. Leung H, Gullick W and Lemoine N: Expression and functional activity of fibroblast growth factors and their receptors in human pancreatic cancer. Int J Cancer 59: 667-675, 1994.

25. Partanen J, Mäkalä T, Eerola E, et al: FGFR-4, a novel acidic fibroblast growth factor receptor with a distinct expression pattern. EMBO J 10: 1347-1354, 1991.

26. Weinstein $M, X u X$, Ohyama $K$ and Deng C: FGFR-3 and FGFR-4 function cooperatively to direct alveogenesis in the murine lung. Development 125: 3615-3623, 1998.
27. Hughes S: Differential expression of the fibroblast growth factor receptor (FGFR) multigene family in normal human adult tissues. J Histochem Cytochem 45: 1005-1019, 1997.

28. Bange J, Prechtl D, Cheburkin Y, et al: Cancer progression and tumor cell motility are associated with the FGFR4 $\operatorname{Arg}(388)$ allele. Cancer Res 62: 840-847, 2002.

29. Wang J, Stockton D and Ittmann M: The fibroblast growth factor receptor-4 Arg388 allele is associated with prostate cancer initiation and progression. Clin Cancer Res 10: 6169$6178,2004$.

30. da Costa Andrade V, Parise H, Hors HP, de Melo Martins PC, Silva AP and Garicochea B: The fibroblast growth factor receptor 4 (FGFR4) Arg388 allele correlates with survival in head and neck squamous cell carcinoma. Exp Mol Pathol 82: 53-57, 2007.

31. Matakidou A, El Galta R, Rudd M, et al: Further observations on the relationship between the FGFR4 Gly388Arg polymorphism and lung cancer prognosis. Br J Cancer 96: 1904-1907, 2007.

32. Shah R, Ibbitt J, Alitalo K and Hurst H: FGFR4 overexpression in pancreatic cancer is mediated by an intronic enhancer activated by HNF1alpha. Oncogene 21: 8251-8261, 2002.

33. Wu X, Lemon B, Li X, et al: C-terminal tail of FGF19 determines its specificity toward Klotho co-receptors. J Biol Chem 283: 33304-33309, 2008.

34. Powers C, McLeskey S and Wellstein A: Fibroblast growth factors, their receptors and signaling. Endocr Relat Cancer 7: $165-197,2000$

35. Kummer C and Ginsberg M: New approaches to blockade of alpha4-integrins, proven therapeutic targets in chronic inflammation. Biochem Pharmacol 72: 1460-1468, 2006.

36. Walsh N, Clynes M, Crown J and O'Donovan N: Alterations in integrin expression modulates invasion of pancreatic cancer cells. J Exp Clin Cancer Res 28: 140, 2009.

37. Korc M: Pancreatic cancer-associated stroma production. Am J Surg 194: S84-S86, 2007.

38. Shaoul E, Reich-Slotky R, Berman B and Ron D: Fibroblast growth factor receptors display both common and distinct signaling pathways. Oncogene 10: 1553-1561, 1995.

39. Raffioni S, Thomas D, Foehr E, Thompson L and Bradshaw R: Comparison of the intracellular signaling responses by three chimeric fibroblast growth factor receptors in PC12 cells. Proc Natl Acad Sci USA 96: 7178-7183, 1999. 